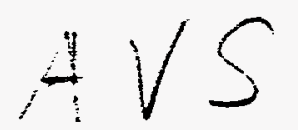

\title{
Mechanisms affecting kinetic energies of laser-ablated materials
}

K. R. Chen ${ }^{1,2}$, J. N. Leboeuf ${ }^{1}$, R. F. Wood ${ }^{1}$, D. B. Geohegan ${ }^{1}$, J. M. Donato ${ }^{1}$, C. L. Liu ${ }^{1}$, and A. A. Puretzky ${ }^{1}$

${ }^{1}$ Oak Ridge National Laboratory, P. O. Box 2009, Oak Ridge, TN 37831-8071, USA

${ }^{2}$ Department of Physics, National ChangHua University of Education, ChangHua, Taiwan 50058, Republic of China

\section{ABSTRACT}

A dynamic source effect was found to accelerate the plume expansion velocity much higher than that from a conventional free expansion model. A self-similar theory and a hydrodynamic model are developed to study this effect, which mav help to explain experimentally observed high front expansion velocity. Background gas can also affect the kinetic energies. High background gas may cause the ablated materials to go backward. Experimentally observed plume splitting is also discussed.

\section{INTRODUCTION}

Laser materials processing techniques are expected to have a dramatic impact on materials science and engineering in the near future and beyond. One of the main laser materials processing techniques is Pulsed Laser Deposition (PLD) for thin film growth. While experimentalists search for optimal approaches for thin film growth with pulsed laser deposition(PLD), a systematic effort in theory and modeling of various processes during PLD is needed.

The quality of film deposited depends critically on the range and profile of the kinetic energy and density of the ablated plume. While it is to the advantage of pulsed laser deposition to have high kinetic energy, plumes that are too energetic causes film damage. We discuss a few mechanisms that affect the kinetic energies.

We have found that, near solid surface, a dynamic source effect can accelerate the plume expansion velocity much higher than that from a conventional free expansion model. A self-similar theory and a hydrodynamic model are developed to study this effect, which may 


\section{DISCLAMMIER}

Portions of this document may be illegible in electronic image products. Images are produced from the best available original document. 
provide an explanation for experimentally observed high expansion velocity. Background gas effects affecting the kinetic energies are also discussed. High background gas may cause the ablated materials to go backward. The physics which may be responsible to the experimentally observed plume splitting is also discussed. The results from the modeling are compared with experimental observations where possible.

\section{DYNAMIC SOURCE EFFECT}

The quality of film deposited critically depends on the range and profile of the kinetic energy and density of the ablated plume[1]. Plumes that are too energetic causes film damage. It is well known $[2,3]$ that the maximum escape velocity of an original stationary gas is $2 c_{s} /(\gamma-1)$ for an unsteady expansion, where $c_{s}$ is the initial sound speed and $\gamma$ is the ratio of specific heats. However, experimental measurements always show that at low laser fluence in which the laser energy absorbed by the plume is negligible, the expansion front is two to three times faster than predicted from unsteady adiabatic expansion with typical vaporization temperatures[3].

We have found a dynamic source effect that accelerates the unsteady expansion significantly faster than predicted from conventional models in the direction perpendicular to the target surface. An effect of dynamic partial ionization that increases the expansion in all directions is also studied. As in previous work[3, 4], we are interested in a laser fluence range high enough for hydrodynamic theory to be applicable, but low enough for the absorption of the laser energy by the plume to be weak so that we can compare it with free expansion models that do not include absorption.

In our model the same material is treated as a dynamic source into the system after $t=0$. For the plume pressure, $P$, below its thermodynamic critical pressure and with low plume viscosity, we may assume that the plume behaves as an ideal gas such that $P=n(1+\eta) k_{B} T$, where $n(T)$ is the density (temperature) of the plume, $\eta$ is the ionization fraction. and $k_{B}$ is the Boltzmann constant. We use Euler's fluid equations to model the plume dynamics and 
the Saha equation to determine the ionization fraction[5]:

$$
\begin{aligned}
\frac{\partial}{\partial t}(n) & =-\frac{\partial}{\partial x}(n v)+S_{n} \delta\left(x-x_{s}\right), \\
m \frac{\partial}{\partial t}(n v) & =-\frac{\partial}{\partial x}\left(P+m n v^{2}\right), \\
\frac{\partial}{\partial t}(E) & =-\frac{\partial}{\partial x}[v(E+P)]+S_{E} \delta\left(x-x_{s}\right), \\
\frac{\eta^{2}}{1-\eta} & =\frac{2}{n} \frac{u_{+}}{u_{o}}\left(\frac{2 \pi m_{e} k_{B} T}{h^{2}}\right)^{3 / 2} e^{-\frac{u_{i}}{k_{B} T}},
\end{aligned}
$$

where $v$ is the plume velocity, $E=m n e+m n v^{2} / 2$ is the plume energy density, $e=(1+$ $\eta)\left(k_{B} T / m\right) /(\gamma-1)+\eta U_{i}$ is the plume enthalpy, $U_{i}$ is the ionization potential, $u_{+}$and $u_{o}$ are the electronic partition functions, $m$ is the mass of the plume atom, $m_{e}$ is the electron mass, $h$ is Plank's constant, $S_{n}=n_{\text {liq }} v_{r s}$ is the density source, $S_{E}=n_{\text {liq }} v_{r s} k_{B} T_{v} /(\gamma-1)$ is the energy source, $n_{l i q}$ is the liquid density, $v_{r s}$ is the recession speed of the target surface due to ablation, and $T_{v}$ is the vapor temperature. Here we take the small Knudsen layer limit, use $v=0$ at the surface, and let $S_{n}$ and $S_{E}$ be constant. Because $c_{s}>v_{r s}$, the surface recession on the plume expansion can be neglected (i.e., $\left.x_{s}=0\right)[6]$.

\subsection{A self-similar theory}

For simplicity and comparision with the free expansion results. our analysis considers the gas to be neutral, which is a good approximation for $T_{v} \ll U_{i}$. With an energy source, the system is non-adiabatic near the surface. We expect self-similar expansion except for early times and a transition region near the surface $(\delta x)$. The self-similar variable is $\xi \equiv x / v_{m} t$, where $v_{m}$ is the maximum expansion velocity. We assume a velocity profile of $v=v_{m}[\alpha+(1-\alpha) \xi]$, where $\alpha$ is determined by the flow properties $(1 \geq \alpha \geq 0)$. We transform the independent variables from $(x, t)$ to $\xi$. From Eqs. (1) and (2) we obtain the density profile $n=n_{\delta}(1-\xi)^{(1-\alpha) / \alpha}$ and the pressure profile $P=n_{\delta} v_{m}^{2} m \alpha^{2}(1-\alpha) /(1+\alpha)(1-\xi)^{(1+1 / \alpha)}$; so the temperature profile is $k_{B} T / m=v_{m}^{2} \alpha^{2}(1-\alpha) /(1+\alpha)(1-\xi)^{2}$. From mass, momentum. and energy conservations, we know the relations of $v_{m}, \alpha, n_{\delta}$, and $T_{\delta}$, which show the maximum expansion velocity scales as $1 / \alpha$. The value $v_{m} / c_{\delta}=4$ for $\alpha=1 / 4$ corresponds to the case of adiabatic expansion with a Knudsen layer[3]. 


\subsection{Numerical hydrodynamic modeling}

The Rusanov scheme[7] is used to solve Euler's equations, Eqs. (1)-(3); the nonlinear calculation of $T$ and $\eta$ is done with the Newton-Raphson method[8]. We use the logarithm of Eq. (4) for numerical stability. The system size is 1,000 spatial grids, $\Delta x$. The initial adaptive size is $10^{-5} \mathrm{~cm}$, which is required for numerical convergence. New vapor is added perturbatively into the first cell near the surface.

The typical physical parameters are as follows. The system is initiated with a uniform background gas with density $n_{b g}=1 \times 10^{10} \mathrm{~cm}^{-3}$ and temperature $T_{b g}=293 \mathrm{~K}$. A constant source of vapor is specified for $6 \mathrm{~ns}$ with a temperature $T_{v}=7000 \mathrm{~K}$, given by the ClausiusClapeyron equation, and the target recession speed is $v_{r s}=1 \times 10^{3} \mathrm{~cm} / \mathrm{s}$, which is typical for the ablation of silicon with laser fluence of a few $J / \mathrm{cm}^{2}$ [9]. We choose the mass of both source and background gas to be 28 a.m.u., a solid density of $5.01 \times 10^{22} \mathrm{~cm}^{-3}$, an ionization potential of $1.3 \times 10^{11} \mathrm{erg}(8.1 \mathrm{eV}), u_{+}=6$, and $u_{0}=15$. We use $\gamma=5 / 3$. Thus, $c_{s}=1.85 \times 10^{5} \mathrm{~cm} / \mathrm{s}$. We note that the conventional free expansion model for no background gas (vacuum) gives a maximum expansion velocity of $5.55 \times 10^{5} \mathrm{~cm} / \mathrm{s}$.

We first study the case without the Saha equation (no ionization; i.e., $\eta=0$ ). We check the profiles of density and velocity at $t=5 \mathrm{~ns}$, as shown on Fig. 1, at which time the expansion is almost steady state. From the simulations. we observe that the expansion develops self-similarly after $0.1 \mathrm{~ns}$. The front position is at $x=0.0069 \mathrm{~cm}$ at $t=5 \mathrm{~ns}$. The maximum expansion velocity at this time is defined to be the ratio of the front position and the time: that is, $v_{m}=1.38 \times 10^{6} \mathrm{~cm} / \mathrm{s}$ or $7.46 c_{s}$. From the slope of the velocity profile, we estimate $\alpha=1 / 14=0.07143$. which gives $v_{\delta}=9.85 \times 10^{4} \mathrm{~cm} / \mathrm{s}$. Thus, $\delta x=6.4 \times 10^{-5} \mathrm{~cm}$. The simulation also shows that $n_{\delta}=4.7 \times 10^{20} \mathrm{~cm}^{-3}$ and $T_{\delta}=3693 \mathrm{~K}$. The analytic profiles are given by $n=n_{\delta}(1-x / 0.0069 \mathrm{~cm})^{13}$ and $v=v_{m} / 14+(13 / 14)(x / 5 \mathrm{~ns})$ from the self-similar theory. The analytic maximum expansion velocity is $7.42 c_{s}$. Also, $n_{\delta}=5.07 \times 10^{20} \mathrm{~cm}^{-3}$ and $T_{\delta}=2836 \mathrm{~K}$. The overall numerical profiles and scalings are in good agreement with the analytical theory. When we use the Saha equation (the more physical case), we find that the maximum velocity is about $40 \%$ higher. It reaches $1.70 \times 10^{6} \mathrm{~cm} / \mathrm{s}$ or $9.2 c_{s}$ at $t=10 \mathrm{~ns}$. 
The higher maximum velocity is an effect due to dynamic partial ionization as a result of increased energy channeled into directed motion. This effect is reduced when the vapor temperature is lower; it gives only about a $6 \%$ increase when $T_{v}=3500 \mathrm{~K}$, for example.

\section{PLUME DYNAMICS IN BACKGROUND GAS}

With background gas, laser-ablated materials can not freely expand. While the collisins between them happen, the materials lose momentum and kinetic energy to the background gas. When the background gas is high, all the ablated materials may encounter at least one collision with the background gas and the hydrodynamic model may be still applicable to this case. However, in a low background gas, the mean-free-path of the ablated materials colliding with the gas may be much longer than the system length such that some of the ablated materials may not encount any collision with the gas at all. We will discuss the two regions respectively.

\subsection{High background gas pressure}

Laser ablation experiments have shown that the plume propagation in high background gas can lead to the stopping of the ablated materials. In some cases the materials can even move backward. and several reflected shocks within the plume are apparent.

With the hydrodynamic modeling, we have simulated the plume dynamics with the following parameters: the recession speed of the solid silicon surface is $100 \mathrm{~cm} / \mathrm{s}$ and lasts for $6 \mathrm{~ns}$ with a vapor temperature of $7000 \mathrm{~K}$, and the background gas density is $6.6 \times 10^{15} \mathrm{~cm}^{-3}$ at room temperature (i.e., the background pressure is $200 \mathrm{mTorr}$ ). Fig. 2 shows the plume dynamics at different times. At $t=10$ ns as shown in Fig. 2(a), the background gas has been snowplowed. Also, the temperature and ionization fraction rise at the shock front. Fig. 2(b) shows that the relative higher pressure at the shock front has split the plume and background. This couples with the rarefaction of the plume to begin pushing the main body

of the plume (second peak) backward and, thus, to slow it down. As a result, the velocity of the second peak is decreased toward zero. By $\mathrm{t}=100 \mu \mathrm{s}$, the velocity has become negative; that is, the second peak moves backward, as indcated in Fig. 2(c). The backward-moving 
plume eventually hits the target surface, rebounds, and moves forward again. The resultant plume splits, as shown in Fig. 2(d). We have also determined the scaling law for the turnover position of the ablated plume. The numerical modeling results show that the turnover position of the ablated plume is inversely proportional to the gas pressure and is proportional to the amount of ablated material.

\subsection{Low background gas}

Plume splitting has been observed experimentally with ion-probe diagnositic in low but finite background gas region. Conceptually, if there are two particles of the same velocity with near-by initial positions going through the background gas, due to Monte Carlo collision, one of them may collide with the gas and be slowed down while the other one may not and continue its original path as in vacuum. So, these two particles split in terms of the detector signal. This concept gives us the foundation to believe that the ablated materils may split in terms of particle distribution in real space. In reality, the plume contains numerous particles. If the background gas is high enough (that is, the mean-free-path is much shorter than the length of interest), the plume is continuous and hydrodynamic model is appropriate. No such plume splitting may be expected. However, if the background gas is low enough for some of the plume particles going through without collision and, but, is high enough for some of them effectively colliding with the background gas, these two groups of the ablated plume may split as in the two particle model. We have developed a model with particle-type collision and hydrodynamic-type plume propagation. The results show that the plume can split under appropriate parameters. More studies is needed for comparision with experimental measurement.

\section{SUMMARY}

We has studied a few mechanisms to affect the kinetic energies of laser-ablated materials. We have treated the laser-ablated material as a dynamic source, which is closer to experimental conditions, rather than as an initial constant source. as is done in free expansion models. It is demonstrated that the dynamic source and partial ionization effects can dramatically 
increase the front expansion velocity, which becomes significantly higher than predicted from a conventional free expansion model. For plume propagation in a high background gas, our results show that the background gas acts on the main body of the rarefying plume, tending to slow it down, and in some cases even results in the backward movement of materials. In a low background gas, we have discussed the possibility of partial plume colliding with the gas for causing plume to split.

\section{ACKNOWLEDGMENTS}

This work was supported by the Oak Ridge National Laboratory (ORNL) Directed Research and Development Funds and the Division of Materials Sciences, U. S. Department of Energy, under contract DE-AC05-84OR21400 with Lockheed Martin Energy Systems, Inc. 


\section{References}

[1] D. B. Chrisey and G. K. Hubler, Pulsed Laser Deposition of Thin Films, John Wiley \& Sons:New York (1994).

[2] L. D. Landau and E. M. Lifshitz, Fluid Mechanics, Addison-Wesley:New York, 357 (1959).

[3] R. Kelly, J. of Chem. Phys. $\underline{92}, 5047$ (1990) and references therein.

[4] R. Kelly, A. Miotello, A. Mele, A. Giardini, J. W. Hastie. P. K. Schenck, and H. Okabe, submitted to Surf. Sci. Also, private communication with R. Kelly.

[5] See, for example, A. Vertes, in 2nd Int'l Conf. on Laser Ablation: Mechanisms and Application-II, edited by J. C. Miller and D. B. Geohegan, AIP Conf. Proc. No. 288, 275 (1994).

[6] G. Weyl, A. Pirri, and R. Root, AIAA J. 19, 460 (1981).

[7] G. A. Sod, J. of Comput. Phys. 27, 1-31 (1978).

[8] W. H. Press, B. P. Flannery, S. A. Teukolsky, and W. T. Vetterling, Numerical Recipes (FORTRAN Version), 267 (1989).

[9] R. F. Wood and G. A. Geist, Phys. Rev. Lett. 57, 873 (1986). 


\section{Figure Captions}

Fig.1. The plume density and velocity profiles at $t=5 \mathrm{~ns}$ from the analytical self-similar theory, numerical hydrodynamic modeling, and conventional free expansion model. Here, we have $\alpha=1 / 14$ and $v_{m}=7.42 c_{s}$ for the analytics, and the initial density in the reservoir $n_{o}=n_{l i q} v_{r s} / c_{s}=2.69 \times 10^{20} \mathrm{~cm}^{-3}$ for the conventional free expansion case.

Fig.2. The profiles of density, pressure, and velocity vs. position at different times: (a) $t=$ $10 \mathrm{~ns},(\mathrm{~b}) \mathrm{t}=1 \mu \mathrm{s}$, (c) $\mathrm{t}=100 \mu \mathrm{s}$, and (d) $\mathrm{t}=500 \mu \mathrm{s}$. 


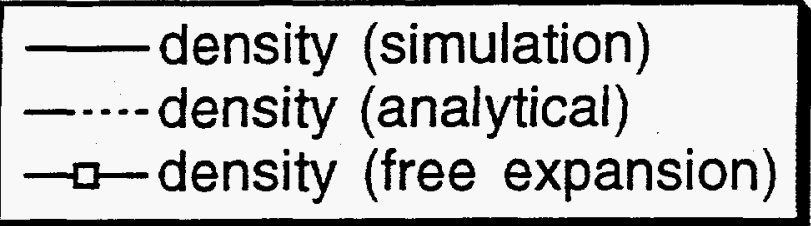

- velocity (simulation)

- - velocity (analytical)

- - velocity (free expansion)

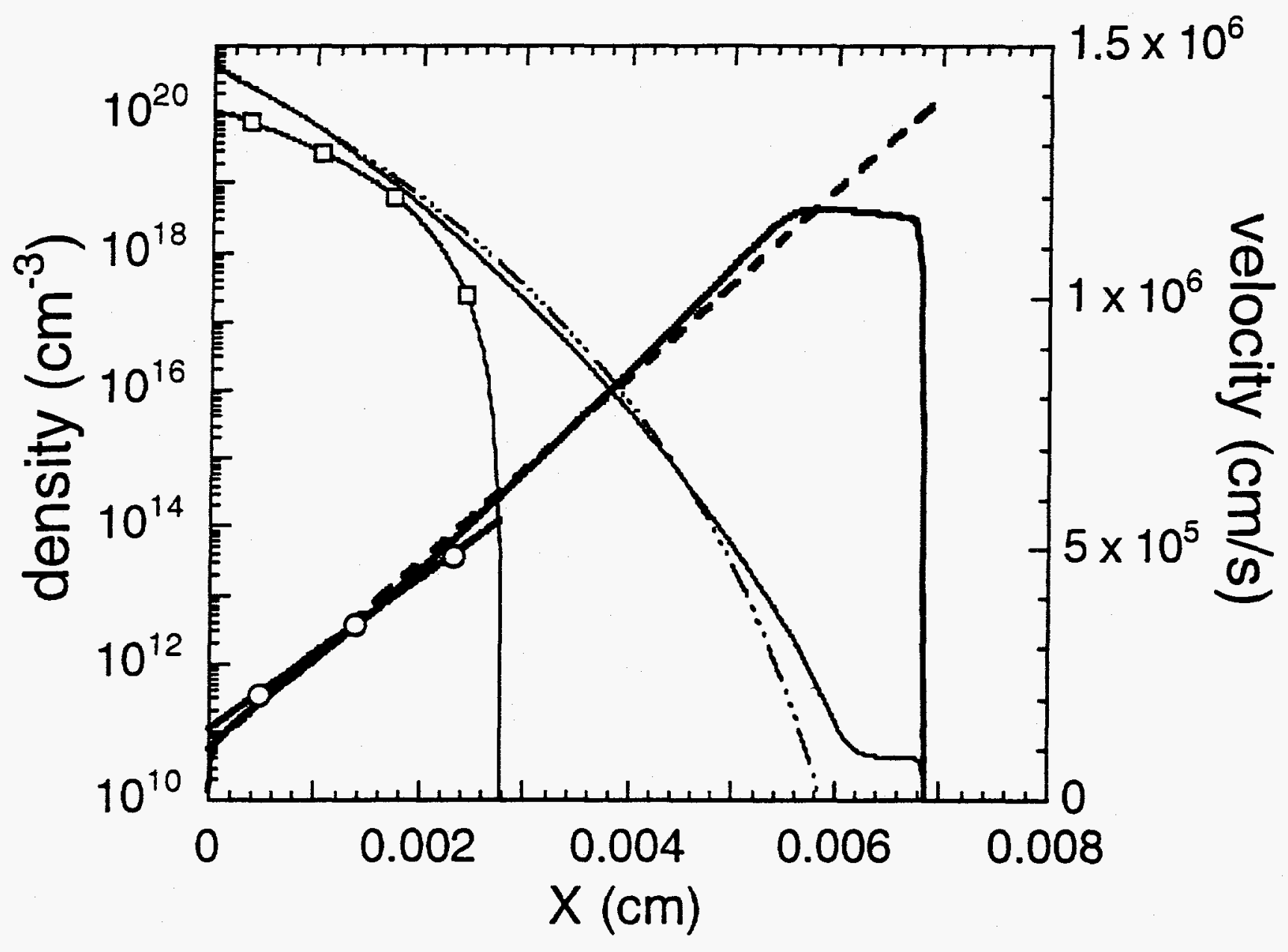

Fig. 1 by Chen, et al. 


\section{DISCLAIMER}

This report was prepared as an account of work sponsored by an agency of the United States Government. Neither the United States Government nor any agency thereof, nor any of their employees, makes any warranty, express or implied, or assumes any legal liability or responsibility for the accuracy, completeness, or usefulness of any information, apparatus, product, or process disclosed, or represents that its use would not infringe privately owned rights. Reference herein to any specific commercial product, process, or service by trade name, trademark, manufacturer, or otherwise does not necessarily constitute or imply its endorsement, recommendation, or favoring by the United States Government or any agency thereof. The views and opinions of authors expressed herein do not necessarily state or reflect those of the United States Government or any agency thereof.

(a)

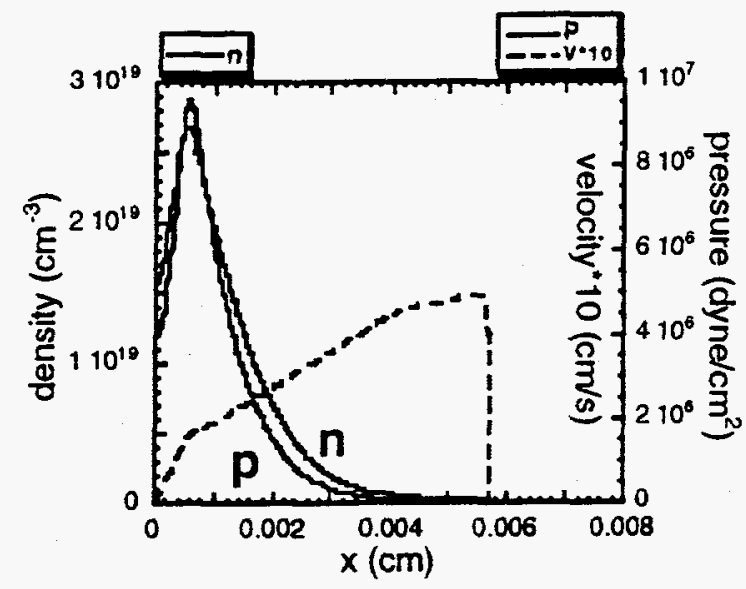

(c)

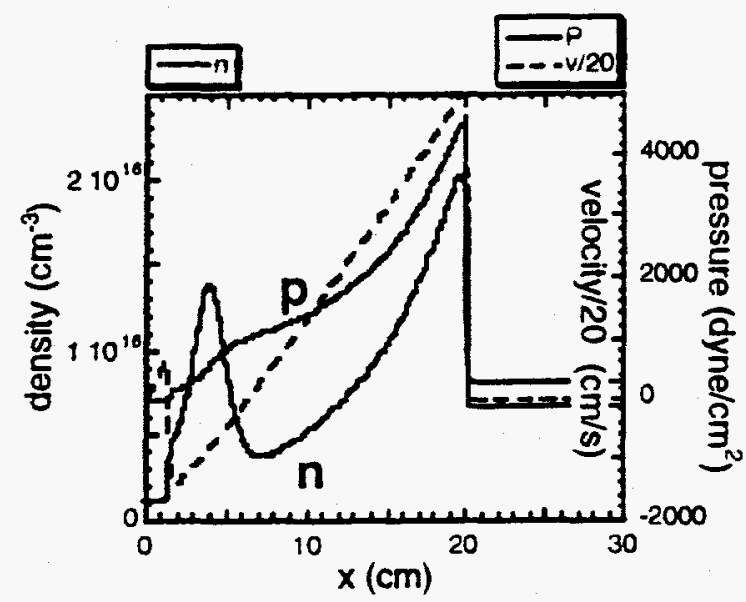

(b)

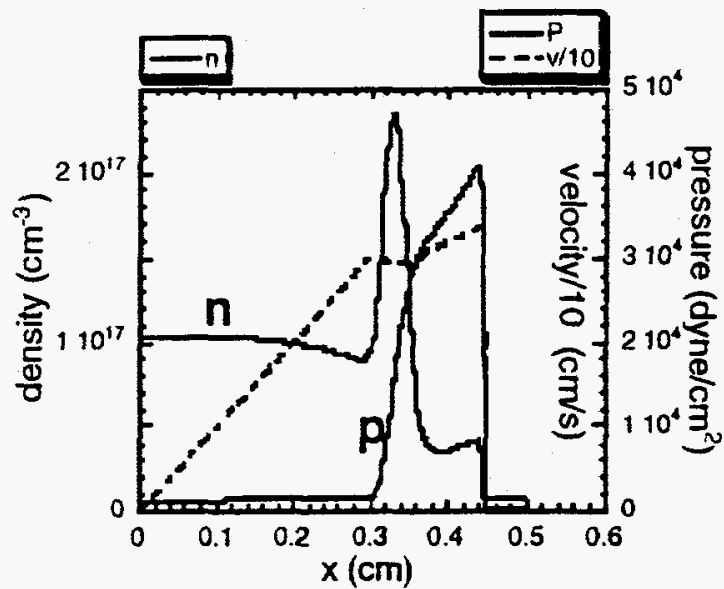

(d)

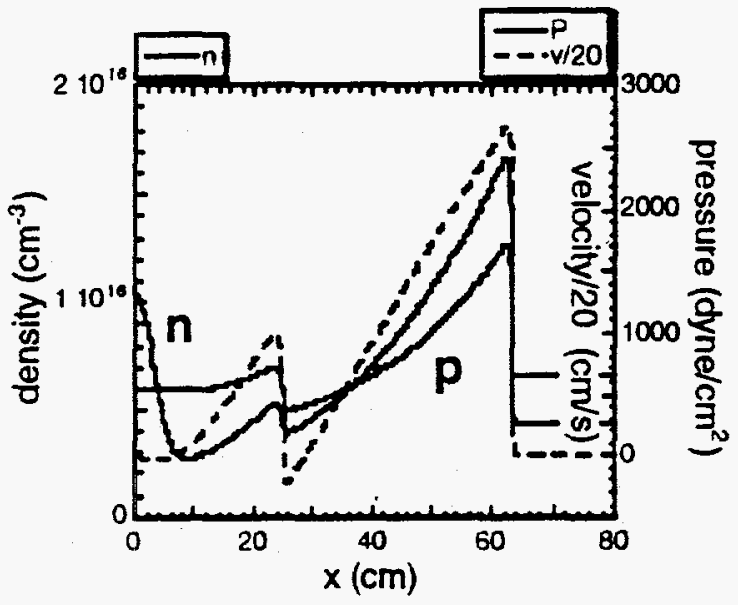

Fig. 2 by Chen, et al. 